\title{
Incidence and risk factors of retinopathy of prematurity in Western India - Report from A Regional Institute of Ophthalmology
}

\author{
Dhaivat Vasavada ${ }^{1,2}$; Sabyasachi Sengupta ${ }^{2}$; Vipul K Prajapati ${ }^{1}$; Shashank M Patel ${ }^{1}$ \\ ${ }^{1} \mathrm{M} \&$ J Western Regional Institute of Ophthalmology, Civil Hospital, Ahmedabad, Gujarat, India \\ ${ }^{2}$ Vitreoretina services, Aravind Eye Hospital, Thavalakuppam, Pondicherry, India
}

\begin{abstract}
Introduction: Retinopathy of prematurity is an important cause of childhood blindness amongst premature babies in developing as well as developed world. Objectives: To report the incidence and risk factors of ROP from a regional institute of Ophthalmology in western India and compare it with the existing scenario. Materials and Methods: This was prospective observational study of babies born at $\leq 34$ weeks of gestational and/or $\leq 1700$ grams birth weight between January 2012 and October 2013. Birth related information viz. $\mathrm{O}_{2}$ administration was recorded in consultation with a neonatologist and babies were evaluated for the presence of ROP. Multivariable logistic regression was used to identify risk factors for any-ROP and severe-ROP requiring treatment. Results: Out of 280 babies screened, any-ROP was seen 54 babies $(19.28 \%)$ out of which, severe-ROP was found in 28 babies(10.29\%).Likelihood of developing any-ROP increased 3 fold $(95 \% \mathrm{CI}=1.1-6.5)$, and severe-ROP by 7 fold $(95 \% \mathrm{CI}=1.6$ -27.5 ), if oxygen therapy as administered. Older gestational age was associated with reduced likelihood of developing severe-ROP (Odds ratio $=0.79,95 \% \mathrm{CI}=0.6-0.9$ ) but not any-ROP. Twenty four babies $(86 \%)$ with severe ROP responded well to laser or Bevacizumab therapy while 4 babies developed retinal detachment. Conclusion: This is the first report quoting only incidence and risk factors of ROP from western India. One in 5 babies develop any-ROP similar to that reported across rest of the country. The incidence of severe-ROP requiring treatment appears to be marginally higher, and treatment outcomes in these eyes are similar to that reported in literature.
\end{abstract}

Keywords: Incidence, western region of India, retinopathy of prematurity, risk factors

\section{Introduction}

Retinopathy of prematurity (ROP) is a well-established disease of premature babies and has emerged as an important cause of childhood blindness. (Kocur et al,2001; Steinkuller et al,1999) The number of blind children approaches 1.5 million with $75 \%$ of

Received: 31/05/16

Accepted: 21/02/17

Corresponding author

Dr. Dhaivat Vasavada

26, Krishna Bungalows, Thaltej-shilaj road,

Ahmedabad, Gujarat - 380059 .

Contact number : 09825909979,09047384607

Email: dha_vas@yahoo.co.in. them residing in the developing world (Gilbert et al,1997). Since the CRYO-ROP cooperative group (Multicenter trial of cryotherapy for retinopathy of prematurity. Preliminary results,1988) established the benefit of treatment when ROP reaches the stage of threshold disease, screening of all premature babies for ROP has become an important measure in preventing childhood blindness. The global initiative for the elimination of avoidable blindness, the WHO vision 2020 
program, targets ROP for prevention of blindness and treatment in an effort to reduce the prevalence of childhood blindness (Gilbert and Foster,2001).

Incidence of ROP was $65.8 \%$ amongst babies with birth weight less than 1251 grams in the CRYO-ROP study cooperative group (Multicenter trial of cryotherapy for retinopathy of prematurity. Preliminary results, 1988) and $68 \%$ in Early Treatment of Retinopathy of Prematurity (ETROP) incidence study (Good et al,2005). The reported incidence of ROP in the West ranges from 21 to $65.8 \%$ (Fielder et al,1992; Darlow,1988). The recently concluded SUPPORT study reported an incidence of 28 $-32 \%$ of severe-ROP in children between 24 -27 weeks gestational age (SUPPORT Study Group of the Eunice Kennedy Shriver NICHD Neonatal Research Network et al, 2010). Azad et al (2003) shows that ROP accounts for $2.3 \%$ to $3.35 \%$ of blindness in all premature children. Previous studies have shown that the incidence of ROP varies from 38\% - 51.9\% among premature and low birth-weight babies (Gopal et al and Charan et al,1995; Varughese et al,2001). Though low birth weight \& gestational age are the primary risk factors leading to the development of ROP, multiple other maternal and fetal risk factors are associated, such as high concentration of oxygen therapy (Penn et al,1995), respiratory distress syndrome, apnea, sepsis, blood transfusions and anemia (Chaudhari et al,2009). The goal for clinicians is to deliver adequate oxygen to the tissue without creating oxygen toxicity in low birth premature babies (Hartnett,2015).

Ours is a tertiary referral eye care center, a governmental organization, designated as a regional institute of Ophthalmology (RIO) for western India in the state of Gujarat and caters to patients from three large states i.e. Gujarat, Rajasthan and large parts of western Madhya Pradesh. The Ophthalmology department is well equipped, has well trained faculty routinely dealing with complex vitreoretinal disorders and is part of a large multispecialty general hospital. As our hospital also boasts of a state - of - the - art neonatal intensive care unit (NICU), it is natural that we are frequently called upon to screen preterm babies for presence of ROP.

There is abundant data from southern, northern and eastern zones of India on the incidence and risk factors for development of any-ROP (Azad et al,2003; Gopal et al and Charan et al,1995; Rao et al, 2013). The Twin - cities ROP project and Karnataka Internet Assisted Diagnosis of Retinopathy of Prematurity program (KIDROP) project are two landmark studies providing extensive insight into situation of ROP in the states of Andhra Pradesh and Karnataka respectively (Jalali et al and Vinekar et al,2014). Although Chaudhari et al (2009) from Pune had studied incidence, risk factors and outcome of laser in ROP. We performed a study to determine the incidence and risk factors for ROP from a regional institute of ophthalmology in western India, which adds to the available Asian data.

\section{Materials and Methods}

This was a prospective, observational study conducted between January 2012 and October 2013 performed at a tertiary referral eye care center, a government organization, designated as a regional institute of Ophthalmology (RIO) for western India in the state of Gujarat that caters to patients from three large states i.e. Gujarat, Rajasthan and large parts of western Madhya Pradesh. The study was approved by the institutional review board and followed the tenets of the declaration of Helsinki and ICH - GCP guidelines. Informed consent was obtained from the parents or legal guardians of all the babies enrolled for the study.

All premature babies born at $\leq 34$ weeks of gestation or weighing less than 1700 grams at birth were enrolled into the study as per 


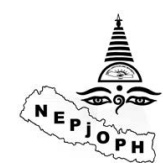

screening guidelines recommended by Jalali et al (2003). Babies born and admitted in the NICU of the hospital or referred from elsewhere within two weeks after birth were included in the study. Those referred with incomplete records, as determined by the attending neonatologist, such as lacking birth weight, gestational weight, APGAR score at birth etc., were excluded from the analysis.

Relevant birth data for all babies satisfying the inclusion criteria were obtained. Parameters recorded were gestational age, gender, birth weight, APGAR score at birth, history of multiple births, mode of delivery (normal vs. Caesarian section vs. assisted), history of fetal distress and/or respiratory distress syndrome (RDS) at birth, meconium staining, and congenital malformations detected at birth. Fetal distress was defined as an abnormal condition of a fetus during gestation or at the time of delivery with altered heart rate associated with hypoxia and acidosis (Thurlow \& Kinsella,2002), RDS was defined as absence of proper surfactant production due to an immature or poorly developed lung (Cole et al,2006). Oxygen administration was performed via hood and continuous monitoring of the partial oxygen pressure $\left(\mathrm{pAO}_{2}\right)$ was done by pulse oximeter. History of oxygen administration immediately after birth or anytime during the stay at NICU and $\mathrm{pAO}_{2}$ at time of birth was recorded as a Yes/No variable.

Other complications during the period of admission such as sepsis (positive blood culture), bradycardia spells (heart rate $\leq$ $100 \mathrm{bpm}$ ), apneic spells (defined by Committee on Fetus and Newborn, 2003 as an episode of cessation of breathing for 20 seconds or longer), anemia, hyperbilirubinemia, phototherapy and blood transfusions were also recorded.

Initial dilated fundus examination was conducted after 2 weeks of birth and if missed, first screening was completed in all the babies
Vasavada D et al

Retinopathy of Prematurity in Western India Nepal J Ophthalmol 2017; Vol 9 (18): 112-120

within 30 days of life. Examination was carried out in the NICU by a single experienced examiner under aseptic precautions using indirect binocular ophthalmoscope, pediatric speculum with lens of +20 Diopters and a scleral depressor under topical anesthesia. Pupils were dilated with $2.5 \%$ phenylephrine and $0.5 \%$ tropicamide. Anterior segment examination was carried out to look for tunica vasculosa lentis, pupillary dilation and media clarity followed by, the posterior pole and peripheral examinations of all clock hours to look for vascularization and presence of the zone and stage of ROP if present.

The main outcome was the occurrence of ROP which was recorded according to International classification of ROP (The Committee for the Classification of Retinopathy of Prematurity,1984). All babies were divided into three groups i.e. No-ROP, Any-ROP and Severe-ROP, which was defined as Type 1 ROP as per the ETROP classification (i.e. zone I, any stage ROP with plus disease; zone I, stage 3 ROP without plus disease; or zone II, stage 2 or 3 with plus disease) and the need for laser/ surgical intervention, or the use of Bevacizumab (SUPPORT Study Group of the Eunice Kennedy Shriver NICHD Neonatal Research Network et al,2010). Any ROP included those with type 1 (severe ROP) as well as those with type 2 ROP (Zone 2 or zone 3 without plus and $\leq$ Stage 2). The classification of ROP was based on ROP status of the worse eye. If both eyes were symmetrically affected, then the status of the right eye was used. Those with severeROP were evaluated at $3-5$ days intervals; those with any-ROP (but less than severeROP) were evaluated weekly, whereas no-ROP babies were reexamined at biweekly intervals till complete vascularization took place. Babies with severe-ROP requiring treatment were either treated with laser photocoagulation to the avascular retina or intravitreal Bevacizumab or both if required. Intravitreal Bevacizumab 
was injected if the eye satisfied the inclusion criteria of the BEAT - ROP study and had stage 3 ROP in zone 1 or zone 2 posterior. Rescue laser was performed for those eyes receiving Bevacizumab if avascular retina persisted after 54 weeks post-conception age (Mintz-Hittner et al, 2011). Eyes developing stage 4 or 5 ROP were referred for vitreoretinal surgery to centers experienced in managing such complicated surgeries.

Outcome measure: Incidence of "any-ROP" and "severe-ROP" and risk factors predictive of ROP occurrence.

\section{Statistical analysis}

Continuous variables were expressed as mean \pm standard deviation (SD) or median and interquartile range (IQR) and categorical variables are presented as percentages. Group differences between eyes with "no-ROP" and "any-ROP" and between "no-ROP" and "severe-ROP" were compared using the Student $t$ test or Wilcoxon rank-sum test for continuous variables and using Chi2 or Fisher exact test for categorical variables. Risk factors predictive of any ROP and severe ROP were assessed using separate Univariate and multivariable logistic regression models. Covariates used were those that are previously established as risk factors of ROP and those that showed more than marginal significance $(p<0.1)$ in univariate analysis. Stepwise forward and backward analysis and nested regression were used to determine the logistic models with best fit.

\section{Results}

Two hundred and eighty babies were included in the study. Out of the 560 eyes, ROP staging was possible in 557 eyes as 3 babies had corneal opacity in 1 eye in which staging could not be done. 247 babies were examined in the "in - house" NICU and 33 referred babies were examined in the outpatients department. Overall, "any-ROP" was seen in 54 babies (19.28\%) out of which severe-ROP was found in 28 babies (10.29\%). All babies showed symmetric presentation in both the eyes. Mean gestational age at time of delivery was $30.6 \pm$ 1.97 weeks (Range $=26-35$ weeks). The birth weight of the babies ranged from 552 to 2400 grams $($ Mean weight $=1639 \pm 414 \mathrm{gm})$. Out of 280 babies, $215(76.78 \%)$ were males and 265 babies $(94.6 \%)$ were products of single pregnancy whereas $15(5.35 \%)$ were products of multiple pregnancies.

Figure 1: Locally weighted scatter-plot smoothing (LOWESS) curves showing the linear relationship between gestational age (X Axis) and birth weight (Y Axis) in babies with and without any-ROP. Beyond 32 weeks, ROP developed in babies with significantly lower birth weight
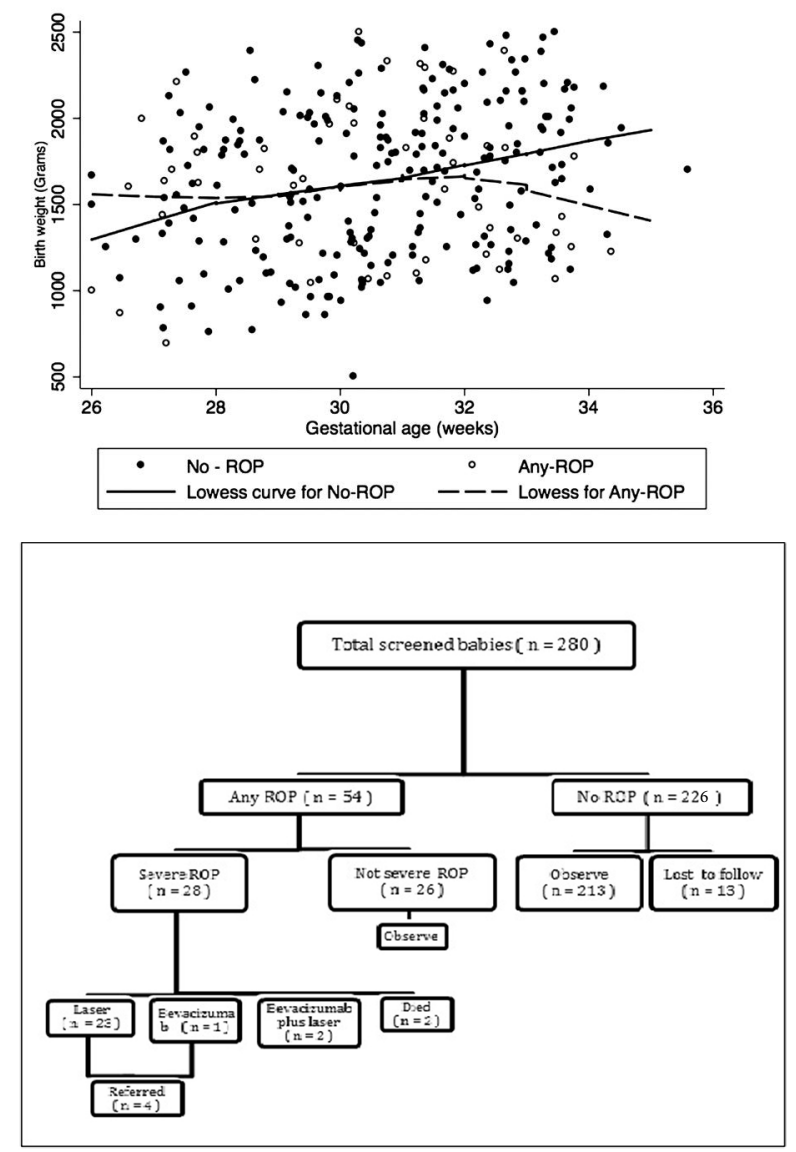

Figure 2: Overall scenario of ROP from a regional institute of Ophthalmology in western India 
Table 1: Comparison of variables between babies with No-ROP vs. Any-ROP and Severe-ROP.

\begin{tabular}{|l|l|l|l|l|l|}
\hline Variable & $\begin{array}{l}\text { No-ROP } \\
(\mathbf{n = 2 2 6})\end{array}$ & $\begin{array}{l}\text { Any-ROP } \\
(\mathbf{n}=\mathbf{5 4})^{*}\end{array}$ & P value $^{*}$ & $\begin{array}{l}\text { Severe ROP } \\
(\mathbf{n = 2 8})\end{array}$ & P Value $^{* *}$ \\
\hline Birth weight (kg) & $1.65 \pm 0.40$ & $1.56 \pm 0.43$ & 0.41 & $1.50 \pm 0.44$ & 0.09 \\
\hline Gest age (wks) & $30.69 \pm 1.92$ & $30.37 \pm 2.17$ & 0.37 & $29.78 \pm 2.28$ & $\mathbf{0 . 0 3}$ \\
\hline Gender (\% males) & $170(75 \%)$ & $45(83 \%)$ & 0.21 & $22(78 \%)$ & 0.70 \\
\hline $\mathrm{Hb}(\mathrm{gm} \%)$ & $11.55 \pm 2.57$ & $11.20 \pm 2.90$ & 0.58 & $10.79 \pm 3.41$ & 0.18 \\
\hline Normal delivery (\%) & $153(68 \%)$ & $35(66 \%)$ & 0.12 & $19(68 \%)$ & 0.80 \\
\hline Oxygen administration (\%) & $140(63 \%)$ & $45(83 \%)$ & $\mathbf{0 . 0 0 4}$ & $25(89 \%)$ & $\mathbf{0 . 0 0 5}$ \\
\hline Fetal distress (\%) & $167(74 \%)$ & $34(63 \%)$ & 0.11 & $20(71 \%)$ & 0.78 \\
\hline Multiple births (\%) & $11(5 \%)$ & $4(7.7 \%)$ & 0.42 & $2(7 \%)$ & 0.54 \\
\hline RDS (\%) & $23(10 \%)$ & $11(20 \%)$ & $\mathbf{0 . 0 4}$ & $6(21 \%)$ & $\mathbf{0 . 0 5}$ \\
\hline Bradycardia spells (\%) & $9(4 \%)$ & $5(9 \%)$ & 0.10 & $3(11 \%)$ & 0.10 \\
\hline Anemia (\%) & $10(4.5 \%)$ & $293.7 \%)$ & 0.58 & $1(3.6 \%)$ & 0.65 \\
\hline Hyperbilirubeinemia (\%) & $74(33 \%)$ & $20(37 \%)$ & 0.55 & $10(36 \%)$ & 0.75 \\
\hline Proven sepsis (\%) & $87(39 \%)$ & $26(48 \%)$ & 0.19 & $12(43 \%)$ & 0.65 \\
\hline Apneic spells (\%) & $23(10 \%)$ & $8(14 \%)$ & 0.33 & $4(15 \%)$ & 0.51 \\
\hline Phototherapy (\%) & $72(32 \%)$ & $20(37 \%)$ & 0.47 & $10(36 \%)$ & 0.68 \\
\hline Transfusion (\%) & $67(30 \%)$ & $19(36 \%)$ & 0.43 & $10(36 \%)$ & 0.51 \\
\hline
\end{tabular}

* P value comparing “Any-ROP to no-ROP”. ** P value comparing "severe-ROP to No-ROP”.

\# Any ROP is inclusive of Severe ROP. Accronyms: ROP - Retinopathy of prematurity, Gest - Gestational, RDS - Respiratory distress syndrome

Table 2: Univariate and Multivariable logistic regression for factors predictive of any-ROP and severe-ROP

\begin{tabular}{|c|c|c|c|c|c|}
\hline \multirow[t]{2}{*}{ Variable } & \multirow[t]{2}{*}{ Interval } & \multicolumn{2}{|c|}{$\begin{array}{l}\text { Any-ROP } \\
\text { Odds ratio }(95 \% \mathrm{Cl})\end{array}$} & \multicolumn{2}{|c|}{$\begin{array}{l}\text { Severe-ROP } \\
\text { Odds ratio }(95 \% \mathrm{Cl})\end{array}$} \\
\hline & & \begin{tabular}{|l|} 
Univariate \\
\end{tabular} & Multivariable & \begin{tabular}{|l|} 
Univariate \\
\end{tabular} & Multivariable \\
\hline Birth weight (kg) & 500 grams & $0.85(0.6-1.2)$ & $0.87(0.6-1.3)$ & $0.65(0.4-1.1)$ & $0.70(0.4-1.2)$ \\
\hline $\begin{array}{l}\text { Gestational } \\
\text { age(wk) }\end{array}$ & 1 week & $0.92(0.8-1.1)$ & $0.88(0.7-1.1)$ & $0.79(0.6-0.9)^{*}$ & $0.79(0.6-0.9)^{*}$ \\
\hline Cesarian section & Vs. normal & $1.18(0.6-2.2)$ & -- & $0.89(0.4-2.1)$ & -- \\
\hline $\mathrm{O}_{2}$ administration & Vs. no $\mathrm{O}_{2}$ & $2.96(1.4-6.4)^{\star}$ & $2.69(1.1-6.5)^{\star}$ & $4.94(1.4-16.8)^{\star}$ & $6.75(1.6-27.5)^{\star}$ \\
\hline Fetal distress & Vs. no distress & $1.66(0.8-3.1)$ & $1.32(0.7-2.6)$ & $1.13(0.5-2.7)$ & $0.87(0.3-2.2)$ \\
\hline Multiple births & Vs. single & $1.62(0.5-5.3)$ & -- & $1.63(0.3-7.8)$ & -- \\
\hline $\mathrm{Hb}(\mathrm{gm} \%)$ & $1 \mathrm{gm} \%$ & $0.95(0.8-1.1)$ & -- & $0.90(0.7-1.1)$ & \\
\hline RDS & Vs. no RDS & $2.26(1.1-5.0)^{*}$ & $1.91(0.8-4.4)$ & $2.40(0.8-6.5)$ & $1.95(0.6-5.8)$ \\
\hline Proven sepsis & Vs. no sepsis & $1.48(0.8-2.7)$ & $1.14(0.6-2.2)$ & 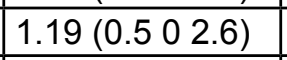 & $0.86(0.4-2.1)$ \\
\hline Hyperbilirubeinemia & Vs. normal & $1.21(0.7-2.2)$ & -- & $1.14(0.5-2.6)$ & -- \\
\hline Bradycardia spells & Vs. no spells & $2.5(0.8-7.8)$ & -- & $3.0(0.7-11.8)$ & -- \\
\hline Apneic spells & Vs. no spells & $1.41(0.6-2.2)$ & -- & $1.47(0.5-4.6)$ & -- \\
\hline
\end{tabular}




\begin{tabular}{|l|l|l|l|l|l|}
\hline Phototherapy & vs. none & $1.26(0.7-2.3)$ & $0.87(0.4-1.7)$ & $1.18(0.5-2.7)$ & $0.79(0.2-2.0)$ \\
\hline Transfusion & Vs. none & $1.28(0.7-2.4)$ & -- & $1.31(0.6-3.0)$ & -- \\
\hline
\end{tabular}

$* \mathrm{P}$ value $<0.05, \mathrm{O}_{2}-$ Oxygen

Table 1 shows a comparative analysis of parameters between babies with no-ROP, any-ROP and severe-ROP. A significantly greater proportion of those with "any-ROP" had received oxygen administration either at birth or during their stay in the NICU compared to those who had no-ROP. Duration of oxygen administration ranged between 4 days and 2 weeks (Median=6 days). Similarly, a greater proportion of babies in the "any-ROP" group had RDS compared to the no-ROP group. We found that those with severe-ROP were almost a week younger at birth and nearly all had received oxygen administration compared to $2 / 3^{\text {rd }}$ of those with no-ROP. In the subgroup of babies born after 32 weeks ( $n=59$ babies), those who experienced any-ROP (11 babies) had significantly lower birth weight than those who didn't experience ROP (1589 $\pm 376 \mathrm{gms}$ vs. $1827 \pm 364 \mathrm{gms}, \mathrm{p}=0.002$ ) (Figure 1). Babies with severe-ROP $(\mathrm{n}=28)$ did not differ from those with ROP not categorized as severe $(n=26)$ in terms of birth weight, $\mathrm{pAO}_{2}$ at birth or oxygen administration. However, these babies were significantly younger $(\mathrm{p}=0.03)$.

Stepwise multivariable logistic regression analysis showed that babies who required oxygen administration had nearly 3 times greater likelihood of developing any-ROP $(p=0.029)$ and almost 7 times greater likelihood of developing severe-ROP $(p=0.008)$ compared to those that didn't receive any oxygen (Table 2 ). In addition to oxygen therapy, older gestational age was significantly associated with reduced likelihood of developing severe-ROP.

Out of the 28 babies with severe-ROP, 2 died $(0.71 \%), 22(78.57 \%)$ were treated with diode laser photocoagulation in one sitting, $1(3.57 \%)$ with two sittings at an interval of two weeks, $1(3.57 \%)$ with a single dose of $0.03 \mathrm{ml}(0.75 \mathrm{mg})$ bevacizumab injection and 2 (7.14\%) with combination of one bevacizumab injection followed by laser photocoagulation. Despite treatment, 8 eyes of 4 babies developed retinal detachment and were referred for timely vitreoretinal surgery. 26 babies ( 48.14 $\%$ ) with ROP Stage 1 or 2 showed mature vascularization on subsequent examination. Remaining 213 babies with no-ROP $(80.71 \%)$ showed mature vascularization within two or three weeks without any intervention while 13 (4.64\%) babies were lost to follow up. Figure 2 shows an overall depiction of the incidence and outcomes of ROP in our study.

\section{Discussion}

We found that 1 in 5 premature babies developed "any-ROP" and 1 in 10 premature babies developed "severe-ROP" in our setting. Oxygen administration was associated with development of ROP and gestational age below 30 weeks was associated with severeROP requiring treatment. In a subset of older babies born beyond 32 weeks gestation, we observed that ROP developed only if they had significantly lower birth weight compared to those who didn't experience ROP.

Many middle-income countries are facing the 'third epidemic' of ROP (Gilbert et al,2005). Jalali et al (2003) estimated that $0.2 \%$ of childhood blindness in India is because of ROP. At present, the prevalence ranges from $0.81 / 1000$ to $1.5 / 1000$ in different states (Jalali et al,2006). Widespread adoption of advanced techniques of assisted reproduction mean these pregnancies are "at risk" and the infants are "precious" leading to premature termination of pregnancy. Though large-scale data is lacking, 


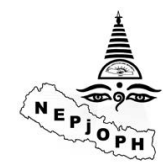

we suspect that the incidence of prematurity is on the rise. On the contrary, better neonatal care, especially judicious oxygen administration, may prevent severe-ROP and vision loss but the burden of disease is likely to rise. Hence, studies are needed to assess the status of ROP periodically.

Our results of the incidence of any-ROP, not requiring treatment and severe-ROP, requiring treatment was comparable to other studies (Rao et al, 2013). A recent report from the KIDROP study in 2015 showed the incidence of any-ROP to be $22.4 \%$ similar to ours, but the incidence of severe-ROP requiring treatment was $3.5 \%$, much lower than our results (Vinekar et al, 2015). In 2009, Chaudhary et al (2009) quoted similar incidence of ROP (22.3\%) in 552 babies with birth weight $<1500 \mathrm{~g}$ and gestation $\leq 32$ weeks. The Twin cities ROP project reported any-ROP in 161 patients out of 643 screened (25\%) (Jalali et al,2014).

In a study by Charan et al (1995), 165 premature babies with a birth weight less than 1700 gms were screened and any-ROP was seen in $47.27 \%$, much higher than what we found. The incidence of severe-ROP was $13 \%$. Similarly Gopal et al (1995) found an incidence of 38\% of any-ROP and 16\% of severe-ROP in his study. Hence, we see a trend towards reduction in the incidence of any-ROP and severe-ROP over two decades. Improved neonatal care and better understanding of the toxic role of oxygen therapy may contribute to this trend. Additionally, greater awareness amongst neonatologists and use of non-ophthalmic personnel to screen for ROP may have led to far better screening which ultimately have reduced the incidence in last decade.

We see similar rates of any-ROP but marginally higher rates $(10 \%)$ of severe-ROP requiring treatment compared to the current standards in India $(4-5 \%)$. Not surprisingly, oxygen therapy and extreme low gestational age were
Vasavada Det al Retinopathy of Prematurity in Western India Nepal J Ophthalmol 2017; Vol 9 (18): 112-120

found to be the most important risk factors for severe-ROP in our patient population.

Most eyes with severe-ROP managed at our center showed good response to treatment with only 4 requiring referral due to unfavorable outcome i.e. retinal detachment. The CRYO-ROP cooperative group (Multicenter trial of cryotherapy for retinopathy of prematurity. Preliminary results, 1988) and ETROP (Early Treatment For Retinopathy Of Prematurity Cooperative Group, 2003) studies reported unfavorable outcome in $21.8 \%$ and $11 \%$ of treated babies respectively. Our treatment results were satisfactory when compared to previous literature and provides credence to the vitreoretina faculty performing ROP screening and treatment.

We found that in a subgroup of babies born after 32 weeks, those who developed ROP had much lower birth weight compared to the majority who didn't develop ROP. Authors have previously reported ROP in infants weighing more than 1250 grams (Gilbert et al, 2005; Vinekar et al, 2007). However, most of these heavier babies are in fact born at or below 32 weeks. The question on risk factors in ROP in older babies, as opposed to heavier babies, is an interesting one and requires further study.

The strengths of our study are its prospective nature, the relatively large sample size reporting the incidence and outcomes of ROP from a previously unreported zone and recording of data directly from the NICU in consultation with a neonatologist. Limitation of this study is the lack of photographic documentation of cases due to unavailability of a Retcam.

In conclusion, the incidence and outcomes of ROP in western India are very similar to that in the developing countries. To the best of our knowledge, this is the first report documenting the situation of ROP spanning three large states. Data from this study will be useful to physicians and commissioners while planning 
programs to prevent childhood blindness across the whole Asia.

\section{References}

An international classification of retinopathy of prematurity. The Committee for the Classification of Retinopathy of Prematurity (1984). Arch. Ophthalmol;102:1130-1134.

Azad RV, Sethi A, Kumar H (2003). Management outcome in prethreshold retinopathy of prematurity. J Pediatr Ophthalmol Strabismus;40:330-334.

Charan R, Dogra MR, Gupta A, Narang A (1995). The incidence of retinopathy of prematurity in a neonatal care unit. Indian $\mathrm{J}$ Ophthalmol;43:123-126.

Chaudhari S, Patwardhan V, Vaidya U, Kadam S, Kamat A (2009). Retinopathy of prematurity in a tertiary care center-incidence, risk factors and outcome. Indian Pediatr;46:219-224.

Cole FS, Nogee LM, Hamvas A (2006). Defects in surfactant synthesis: clinical implications. Pediatr. Clin. North Am;53:911927.

Committee on Fetus and Newborn. American Academy of Pediatrics (2003). Apnea, sudden infant death syndrome, and home monitoring. Pediatrics;111:914-917.

Darlow BA (1988). Incidence of retinopathy of prematurity in New Zealand. Arch. Dis. Child;63:1083-1086.

Early Treatment For Retinopathy Of Prematurity Cooperative Group (2003). Revised indications for the treatment of retinopathy of prematurity: results of the early treatment for retinopathy of prematurity randomized trial. Arch. Ophthalmol;121:1684-1694.

Fielder AR, Shaw DE, Robinson J, Ng YK (1992). Natural history of retinopathy of prematurity: a prospective study. Eye (Lond);6:233-242.

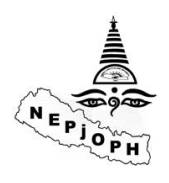

Gilbert C, Rahi J, Eckstein M, O’Sullivan J, Foster A (1997). Retinopathy of prematurity in middle-income countries. Lancet;350:12-14.

Gilbert C, Foster A (2001). Childhood blindness in the context of VISION 2020-the right to sight. Bull World Health Organ; 79:227-232.

Gilbert C, Fielder A, Gordillo L, et al (2005). Characteristics of infants with severe retinopathy of prematurity in countries with low, moderate, and high levels of development: implications for screening programs. Pediatrics;115:e518-525.

Good WV, Hardy RJ, Dobson V, Palmer EA, Phelps DL, Quintos M, Tung B. Early Treatment for Retinopathy of Prematurity Cooperative Group, (2005). The incidence and course of retinopathy of prematurity: findings from the early treatment for retinopathy of prematurity study. Pediatrics;116:15-23.

Gopal L, Sharma T, Ramachandran S, Shanmugasundaram R, Asha V (1995). Retinopathy of prematurity: a study. Indian J Ophthalmol;43:59-61.

Hartnett ME (2015). Pathophysiology and mechanisms of severe retinopathy of prematurity. Ophthalmology;122:200-210.

Jalali S, Anand R, Kumar H, Dogra MR, Azad R, Gopal L (2003). Programme planning and screening strategy in retinopathy of prematurity. Indian J Ophthalmol;51:89-99.

Jalali S, Matalia J, Hussain A, Anand $R$ (2006). Modification of screening criteria for retinopathy of prematurity in India and other middle-income countries. Am. J. Ophthalmol;141:966-968.

Jalali S, Anand R, Rani PK, Balakrishnan $D$ (2014). Impact of the day-30 screening strategy on the disease presentation and outcome of retinopathy of prematurity. The Indian twin 
NE

cities retinopathy of prematurity report number 3. Indian J Ophthalmol;62:610-614.

Kocur I, Kuchynka P, Rodný S, Baráková D, Schwartz EC (2001). Causes of severe visual impairment and blindness in children attending schools for the visually handicapped in the Czech Republic. $\mathrm{Br} \mathrm{J}$ Ophthalmol;85:1149-1152.

Mintz-Hittner HA, Kennedy KA, Chuang AZ, BEAT-ROP Cooperative Group (2011). Efficacy of intravitreal bevacizumab for stage $3+$ retinopathy of prematurity. $\mathrm{N}$. Engl. J. Med.;364:603-615.

Multicenter trial of cryotherapy for retinopathy of prematurity. Preliminary results. Cryotherapy for Retinopathy of Prematurity Cooperative Group (1988). Arch. Ophthalmol;106:471-479.

Penn JS, Henry MM, Wall PT, Tolman BL (1995). The range of $\mathrm{PaO} 2$ variation determines the severity of oxygen-induced retinopathy in newborn rats. Invest. Ophthalmol. Vis. Sci.;36:2063-2070.

Rao KA, Purkayastha J, Hazarika M, Chaitra R, Adith KM (2013). Analysis of prenatal and postnatal risk factors of retinopathy of prematurity in a tertiary care hospital in South India. Indian J Ophthalmol;61:640-644.

Steinkuller PG, Du L, Gilbert C, Foster A, Collins ML, Coats DK (1999). Childhood blindness. J AAPOS;3:26-32.

SUPPORT Study Group of the Eunice
Kennedy Shriver NICHD Neonatal Research Network. Carlo WA, Finer NN, Walsh MC, et al (2010). Target ranges of oxygen saturation in extremely preterm infants. N. Engl. J. Med;362:1959-1969.

Thurlow JA, Kinsella SM (2002). Intrauterine resuscitation: active management of fetal distress. Int J Obstet Anesth;11:105-116.

Varughese S, Jain S, Gupta N, Singh S, Tyagi V, Puliyel JM (2001). Magnitude of the problem of retinopathy of prematurity. experience in a large maternity unit with a medium size level-3 nursery. Indian J Ophthalmol.;49:187-188.

Vinekar A, Dogra MR, Sangtam T, Narang A, Gupta A (2007). Retinopathy of prematurity in Asian Indian babies weighing greater than 1250 grams at birth: ten year data from a tertiary care center in a developing country. Indian J Ophthalmol.;55:331-336.

Vinekar A, Gilbert C, Dogra M, et al (2014). The KIDROP model of combining strategies for providing retinopathy of prematurity screening in underserved areas in India using wide-field imaging, tele-medicine, non-physician graders and smart phone reporting. Indian J Ophthalmol;62:41-49.

Vinekar A, Jayadev C, Mangalesh S, Shetty B, Vidyasagar D (2015). Role of tele-medicine in retinopathy of prematurity screening in rural outreach centers in India - A report of 20,214 imaging sessions in the KIDROP program. Semin Fetal Neonatal Med.

\section{Source of support: nil. Conflict of interest: none}

